\title{
Sobre la naturaleza de la investigación lingüística: antecedentes y perspectivas*
}

\author{
FÉLIX Quesada CAstiliL \\ universidad Nacional Mayor de San Marcos
}

\section{$\&$}

\section{Restmen}

El presente artículo tiene por dojeto propiciar una reflexión sobre las diferentes propuestas de explicación acerca de la naturaleza del lenguaje. Se hace un somero repaso sobre los antecedentes de la lingüística para concluir en una reflexión en tomo a las perspectivas actuales.

En vista de que la lengua constituye un sistema subyacente que genera las expresiones del comportamiento verbal, una teoría lingüística adecuada exige un enfoque intemalista. Según esta perspectiva, el ser humano es capaz de hablar una lengua por cuanto está dotado de una facultad del lenguaje, o de un órgano de la mente/cerebro, y esta dotación genética explica el aprendizaje de una lengua y la capacidad de usar la lengua, debido a la conexión entre el módulo de la facultad del lenguaje (estado asumido al hablar una lengua cualquiera) y los sistemas o módulos de la performance. Este avance alcanzadb por el desarrollo de la teoría generativa actual ha conducido a proponer la biolingüística como ciencia del lenguaje, lo que conduce a integrar la lingüística con las denás ciencias del cerebro, debil itándose su vínoulo con las ciencias sociales.

Palabras claves: Enfoque intemalista, facultad de lenguaje, mente/cerebro, biolingǘstica.

\section{Abstract}

The present article aims at encouraging reflection on the different explanation proposals about the nature of language. A shallow review is made on linguistics background to conclude in a reflection on the current perpectives. 
In view of the fact that language constitutes an underlying system that generates the expressions of verbal behavior, an appropriate linguistics theory demands an intemalist approach. According to this perspective the human being is able to speak a language since he or she is endowed with language faculty, or with an organ of the mind/brain, and this genetic endowment explains the leaming of a language and the ability to use the language, due to the connection between the module of the language faculty (state assumed when speaking any language) and the performance systems or modules. This step forward reached by the development of the arrent generative theory has led to propose biolinguistics as a science of language, what leads to integrate linguistics with the other sciences of the brain, weakening its bond with the social sciences.

Key words: Intemalist approach, language ability, mind/brain, biolinguistics.

\section{Introducción}

Hi presente artículo intenta caracterizar el avance en el conocimiento lingüística de los últimos 20 años. Sin embargo, las diferentes propuestas teóricas sobre las lenguas naturales se han manifestado en el desarrollo de los estudios lingüísticos desde hace un siglo y medio.

Los diferentes enfoques han contribuido, desde su propia perspectiva, en el entendimiento de la naturaleza del lenguaje, su aprendizaje y su rol en la organización y funcionamiento de la sociedad.

El desarrollo de la teoría lingüística ha reflejado las posiciones questas presentes en la actividad científica: (i) el quehacer científico como una colección de datos y las generalizaciones a partir de la observacióny el análisis de éstos; y (ii) la investigación que considera a los datos como manifestaciones fragmentarias e imprecisas de leyes o principios subyacentes.

En esta ocasión no elucidaremos las pasiciones discrepantes acerca de la inclusión de la lingüística en el campo de las ciencias humanas (dado que abarca el conocimiento del hombre y su conducta) o en la esfera de las ciencias sociales (en cuanto elemento de comunicación entre los miembros del grupo o la sociedad) .

La consideración no controversial es que el lenguaje humano posee dos dimensiones: la caracterización del individuo o ser humano y su 
función en la interacción social. Estas dos dimensiones son esenciales para develar las propiedades de las lenguas naturales.

El objetivo fundamental de nuestra aproximación es presentar el enfoque «naturalista» o intemalista como una altemativa interesante del paradigma generativo (Chomsky 1998 y 2002) .

\section{Situación actual sobre el conocimiento del lenguaje y las lenguas}

\section{El significado de la capacidad de hablar}

Ia capacidad de hablar, es decir, la facultad del lenguaje es una «propiedad de la especie», con escasa variación entre los seres humanos y sin que exista nada análogo en otros seres biológicos (Chomsky 1998:13).

Ia trascendencia del lenguaje y las lenguas para el ser humano, así como para la organización y el desarrollo de las sociedades, ha subyugado a filósofos y científicos sociales y, particularmente, a los lingüistas. La elucidación de la naturaleza del lenguaje y el entendimiento de la conducta lingüística como parte de su funcionamiento, han conducido a la fundación y desarrollo de la ciencia lingüristica, cuya importancia se refleja en el auge sin precedentes del enfoque lingüístico en las ciencias sociales. De tal forma que en la filosofía de la segunda mitad del siglo XX, la lingüística adquiere primacía; es decir, el tema esencial y predilecto ha sido la reflexión sobre el lenguaje, lo que se ha denominado el giro lingüristico.

\section{Caracterización del lenguaje camo objeto de estudio}

A través del desarrollo de la lingüística se ha manifestado una controversia sobre qué se entiende por lenguaje y cómo debería estudiarse el lenguaje. Éste puede ser caracterizado como un objeto «multidimensional», pues revela diferentes dimensiones: el aspecto formal (que incluye lo estructural y relacional de la lengua), una dimensión neuronal, un estrato psicológico y la esfera de lo social. Estos diferentes aspectos del lenguaje humano han conducido a varias líneas de investigación desole el punto de vista formal, neurobiológico, 
psicolingüístico y sociolingüístico. Asimismo, se han analizado los vínculos entre el lenguaje y la cultura, así como se ha estudiado el lenguaje desde la perspectiva del desarrollo histórico.

La historia de la lingüística muestra que las diferentes teorías han abordado el aspecto estructural que es el dojeto de la teoría gramatical o lingüística «pura». Para el lingüista puro el lenguaje es un «sistema» de símbolos que posee ciertas propiedades sintácticas, semánticas y fonológicas (Bunge 1983:15).

Además de la teoría gramatical, el lenguaje natural ha sido objeto de los enfoques de la psicolingüística, la sociolingüística, la etnolingüística y, recientemente, la neurolingüística. Se observa que el enfoque de cada aspecto del objeto (a saber, el lenguaje) es parcial, pero cada uno constituye una contribución al entendimiento del lenguaje: cada una de las perspectivas define su dbjeto de investigación a la luz de lo que le interesa remarcar y no se puede negar que ha habido algunos nexos entre los diversos enfoques.

Estos diferentes enfoques y sus logros requieren de una teoría que, sin menoscabar ninguna perspectiva, se constituya en una concepción totalizadora (u holística) sobre el lenguaje. Es decir, se trata de construir una teoría sintetizadora, que no excluya los diferentes enfoques exigidos por la rica naturaleza de las lenguas.

\section{Fil desarrollo de la lingüística y el conocimiento del lenguaje}

La aplicación de la teoría de Kuhn (1962) al desarrollo de la lingüística, nos conduce a considerar los siguientes «paradigmas» en la progresión de la lingüística desde la antigüedad greco-ramana hasta el presente: gramática tradicional, la lingüística histórico-comparativa, el estructuralismo, la gramática generativa, la gramática funcional y la lingüística o gramática del texto (Jacobsen 1986) .

Cada teoría o paradigma ha tratado de desentrañar la naturaleza del lenguaje y las lenguas. Para lograr el objetivo, se han propuesto varias premisas teóricas, a partir de las cuales se han inferido una serie de métodos en el estudio de las lenguas.

En los siguientes apartados vamos a explicitar algunos enfoques de la historia de la lingüística, sin pretensión de exhaustividad. De 
manera que nos centraremos en los elementos que definen a cada teoría o «paradigma». Luego, en armonía con el objetivo de nuestro artículo, nos concentraremos en la versión final (Chomsky 1998 y 2002) de la gramática generativa, cuyo enfoque se caracteriza como un nuevo paradigma dentro del desarrollo de esta teoría.

\section{El modelo de los neogramáticos}

Durante el siglo XIX el estudio de las lenguas busca la constitución de la lingüŕstica como disciplina científica. Se propone caracterizar la naturaleza de la lengua como una realidad mutable, es decir, como un cont inurm que va generando gradualmente la diversidad de lenguas naturales. Ia realidad dinámica del lenguaje se explicita de acuerdo con ciertas leyes que rigen los cambios lingüísticos.

Los neogramáticos, directos herederos de los comparatistas, definieron el objeto de la ciencia en gestación, como una realidad histórica constituida por la commidad lingüristica actual sin dejar de lado las raíces del pasado. El enfoque neogramático louscaba identificar los principios que determinaban la evolución de las lenguas. Asimismo, los neogramáticas establecieron los principios teóricas y metooblógicas de la investigación del cambio lingüristico. Con los comparatistas y los neogramáticos se instituye el cambio lingüŕstico como una de las características esenciales de las lenguas naturales, de donde se deriva la impasibilidad científica de una lingürística que no fuera histórica. Los aportes empíricos y teórico-metodológicos de los neogramáticos han constituido una línea de desarrollo de la lingüística del siglo XX (la lingüŕsticahistórica) .

\section{터 estructuralismo}

La búsqueda de la identificación del dojeto de la lingüística surge con las neogramáticos y se consolida con Saussure (2002), al definir la noción de lengua como una «realidad histórica» y una «totalidad organizada». Saussure, considerado un hito en la historia de la lingüŕstica, propuso la construcción de una ciencia sincrónica de la lengua en oposición al estudio del habla y concibió la lengua como un 
«sistema autónomo» que puede ser estudiado en sí mismo (Marcuschi y Martins Salamão 2004: 15-16) . Sobre la base de esta concepción teórica definió un «programa de investigación», a partir del cual se derivaron diversas teorías lingüísticas contemporáneas: estructuralismo, funcionalismo y la lingüística del texto.

El apogeo alcanzado por el estnuctural ismo y la rigurosidad analítica en la elaboración de sus gramáticas condujeron a que fuera aplicado a las otras ciencias (como la antropología) . Gracias a esta fertilidad, la lingüŕstica fue considerada como modelo y recibió la denominación de «ciencia piloto».

El enfoque estructuralista aplicado en Estados Unidos (cuyos fundamentos teóricos están explicitados en autores como Bloomfield) , consiste en la generalización de constructos gramaticales a partir de los datos. Así la actividad científica se concibe como una recolección de datos, como una actividad clasificatoria y de generalización. En oposición a esta práctica de la ciencia, surge la teoría generativa de raigambre chomskiana que concibe la ciencia lingüística como una teorización sobre las lenguas en el marco de la epistemología estándar: esto es, las doservaciones no son los pilares en la construcción de las teorías, sino son evidencias de una realidad subyacente que explica la conducta lingüística de los miembros de una comunidad. En este contexto, la gramática generativa significó el surgimiento de un nuevo paradigma constituido por un conjunto de hipótesis incompatibles con las anteriores corrientes. El muevo paradigma o enfoque construyó nuevos conocimientos y resolvió problemas que la teoría estructuralistafuncionalista fue incapaz de resolver.

\section{Ell lenguaje, ¿herramienta commicativa o espejo de la mente?}

En los últimos 40 años se han enfrentado dos posiciones acerca de la naturaleza del lenguaje: Una fuerte corriente de opinión considera al lenguaje, esencialmente, como un medio de comunicación y se ha constituido el habla o uso como objeto de la teoría lingüística. Del lado opuesto, Chomsky y sus seguidores han caracterizado al lenguaje como el espejo de la mente que, de manera accesoria, se emplea como un medio de comunicación. En consecuencia, la teoría lingüística 
chomskiana ha buscado elucidar prioritariamente la competencia y la gramática generativa se ha configurado como un enfoque mentalista que lussca caracterizar el sistema cognitivo que constituye la gramática de una lengua.

\section{Ell paradigma generativo}

La gramática generativa, en cuanto teoría de una lengua particular y de las lenguas naturales, surgió en el contexto de lo que ha menudo se llama la «revolución cognitivista» de los años 50 y fue un factor importante en el desarrollo de ésta. Implicó un importante cambio de perspectiva: del estudio del comportamiento y sus productos (enfoque conductista), se pasó al estudio de los mecanismos internos que participan en el pensamiento y la acción. Ia perspectiva cognitivista no considera el comportamiento y sus productos como objeto de estudio, sino como datos que pueden aportar evidencia respecto de los mecanismos internos de la mente y los modos en que estos mecanismas funcionan al ejecutar acciones e interpretar la experiencia. Las propiedades y problemas que fueron el foco de atención para la lingüística estructural tienen un lugar, pero como fenómenos que hay que explicar junto con innumerables otros en términos de los mecanismos internos que generan las expresiones verbales (Chomsky 1998) . Este enfoque es mentalista y constituye la reinterpretación de la primera revolución cognitiva del sigloXVII y XVIII, la cual fue parte de la revolución científica que cambió de manera radical nuestra comprensión del mundo.

Ell dbjetivo de la teoría lingüística es explicar la facultad del lenguaje, esto es, su estado inicial y los estados que asume. Ia facultad del lenguaje puede ser considerada como «órgano del lenguaje que es como otros órganos, y en cuanto a su naturaleza está genéticamente determinada». El objeto de la investigación es el estado inicial genéticamente determinado de la facultad del lenguaje. La teoría de la gramática, a través de su desarrollo, va precisando en qué consiste el estado inicial y ese conocimiento es fundamental para erigir una imagen científicamente plausible de la naturaleza humana. Así, la finalidad de Chomsky (1986) es estudiar un objeto real en el mundo 
natural -el cerebro, sus estados y sus funciones-, de modo que gradualmente el estudio de la mente pueda integrarse con el enfoque propio de las ciencias biológicas. Por tanto, la teoría gramatical tiene por objeto el conocimiento lingüístico representado en la mente/ cerebro. Este enfoque permite construir una teoría que satisface plenamente las condiciones de una buena teoría lingüística, esto es, una teoría con adecuación descriptiva y adecuación explicativa (en el sentido de que postula principios de gramática universal) .

La gramática de una lengua natural satisface la adecuación descriptiva si da cuenta de las propiedades de las lenguas, del saber tácito de los hablantes competentes en la lengua. De otro lado, si se quiere cumplir con el requisito de la adecuación explicativa, la teoría del lenguaje debe mostrar que cada lengua particular debe ser derivada de un estado inicial uniforme bajo condiciones altamente restringidas. Por tanto, la concepción de la gramática que se establece a partir de la confrontación del estado inicial con los resultados de la experiencia propone una teoría que permite explicar los fenómenos complejos del sistema cognitivo representados en la mente/cerebro.

\section{Concepción naturalista del lenguaje}

El modelo de la teoría estándar (Chomsky 1965), luego de casi una década de confrontación con las lenguas, resultó incapaz de dar cuenta de una serie de aspectos empíricos en la construcción de la gramática universal y de las gramáticas de las lenguas, de la adquisición de la lengua y del uso del lenguaje. Esta inadecuación de la inicial teoría generativa condujo a la búsqueda de un nuevo paradigma, para lo cual se construyeron varios modelos. Desde Chomsky (1976) se empezaron a vislumbrar los problemas y misterios que sugería la indagación sobre el lenguaje humano y que en las últimas dos décadas se consideraron como los temas centrales cuya elucidación contribuiría a la explicación de la naturaleza del lenguaje.

La lingüística generativa actual está dominada por la teoría de Principios y Parámetros (Chomsky 1986) y se apoya sobre las bases del programa minimalista (Chomsky 1995). En los diez últimos años se han dado nuevos tipos de evidencia para las indagaciones hechas en 
el muevo marco generativo. De otro lado, existe ahora la posibilidad de formular preguntas cuyo planteamiento antes no era posible y que constituían misterios acerca de la naturaleza del lenguaje. Si bien es cierto que aún no tenemos respuestas para todas ellas, la capacidad y pertinencia de formularlas es por sí mismo un avance excitante (como nos ilustra Smith en su proemio a Chomsky 2002) .

La primera de estas preguntas se refiere a la hipótesis de que una parte sustancial del conocimiento del lenguaje está genéticamente determinada (o es innata), por cuanto las evidencias indican claramente que sólo los seres humanos adquieren un sistema simbólico como el lenguaje.

En Chomsky (2002) hay nuevas soluciones a las confusiones tradicionales y se abren nuevas perspectivas de interés general, desde el problema del llamado dualismo cuerpo/mente hasta el tema de la unificación de la ciencia.

Los problemas y misterios del lenguaje, que se empezaron a vislumbrar en Chomsky (1976), han sido reexaminados en Chomsky (1998) y Chomsky (2002) con el aporte de nuevas propuestas e indagaciones. De ese modo se configura un nuevo paradigma que se orienta a la explicación del lenguaje y de la mente en términos del natural ismo científico, de donde se derivan la intenpretación intemalista de la facultad del lenguaje y la consideración del lenguaje como un dojeto biológico.

Estos aspectos del último paradigma generativo (Chomsky 1995, 1998, 2002) serán sucintamente expuestos a continuación. Nuestras reflexiones tratarán de perfi l ar el enfoque naturalista, tal como se asume y se aplica en las más recientes indagaciones chomskianas.

\section{Ia perspectiva internalista}

El nuevo paradigma aboga por una visión «intemalista» de la facultad del lenguaje humano, con lo que se aparta de las aproximaciones de los filósofos del lenguaje. Ia tradición filosófica se ha concentrado en el lenguaje como un «constructo público» del cual las individuas tienen conocimiento parcial. Esta visión se preocupa sustancialmente de la relación entre el lenguaje y la realidad extema: la relación entre la 
palabra y el mundo que ha dado origen a las teorías estándares de la semántica referencial.

En oposición a esta consideración, Chomsky (2002) defiende el punto de vista de que el conocimiento del lenguaje es necesariamente individual y, como tal, intemo a la mente/cerebro. Esta interpretación conduce a dos importantes consecuencias:

a El estudio apropiado del lenguaje debe apuntar al constructo mental referido como «lenguaje-I», como propiedad intema de un individuo.

b) El enfoque internalista concibe la facultad del lenguaje como un componente de la mente/cerebro, es decir, de naturaleza neuroanatómica. En ese sentido, el lenguaje puede ser caracterizado como una estructura biológica.

La última consecuencia tiene gravitación, por cuanto implica un radical cambio de marcha respecto del anterior enfoque generativo. En efecto, hasta las versiones del programa generativo de la década del setenta se suponía que los seres humanos podían hablar y entender una lengua en virtud de la disposición de la competencia gramatical de naturaleza psicológica, razón por la cual se decía que la lingüística pertenecía a la psicología cognitiva. Para entender la competencia gramatical se postulaba la existencia de un «sistema de reglas que generan y relacionan ciertas representaciones mentales» de forma y significado. Dichas reglas «operaban» en función de determinados principios generales.

A partir de Chomsky (1986), se comienza a estudiar con más profundidad un tema tocado ya por Eric Lenneberg (1967) : los «fundamentos biológicos de las capacidades lingüŕsticas humanas». Gracias al conocimiento de la neurobiología, surge la renuencia a considerar que las teorías lingüísticas ofrezcan sólo hipótesis psicológicas y se inicia una tendencia a entenderlas e interpretarlas en la forma que se acostumbra en las ciencias naturales. Esta tendencia entre los científicos del lenguaje condujo a que Chomsky (1988) considerase que una teoría lingüística debe dar cuenta de las propiedades formales abstractas, de la adquisición y del uso del lenguaje. En consecuencia, una teoría lingüŕstica adecuada debería proponer respuestas como resultado de la indagación lingüística a los siguientes problemas o interrogantes: 
(i) Qué constituye el conocimiento lingüŕstico.

(ii) Cómo se adquiere dicho conocimiento.

(iii) Cómo entra en acción el conocimiento en la comprensión y el uso del lenguaje.

Es decir, toda teoría gramatical debe elucidar el conocimiento de la gramática y el uso del lenguaje. Para responder a estas interrogantes, se hacía necesario adoptar el enfoque naturalista que proponía que la facultad del lenguaje constituía un componente interno de la mente/ cerebro y, por tanto, de naturaleza neuroanatómica o física.

Esta propuesta tuvo como antecedentes a Strawson (1977) que es un claro intento de explicar en términos «naturalistas los dominios de la mente» (Lorenzo 2001: 72) y, más tarde, a Gerald Edelman (1992) . Edelman sostiene que el estudio de la mente sólo alcanza el carácter científico «en el punto en el que las explicaciones consiguen dar cuenta de la relación entre lo psicológico (es decir, el relato funcional o abstracto de las capacidades mentales) y lo fisiológico (o sea, la plena justificación neuroanatómica de aquellas) » (Lorenzo 2001: 73). De acuerdo con la posición de Edelman, se desprende que cualquier explicación de la «mente sin biología» cae en descrédito y, por tanto, constituye una crítica a los modelos generativistas anteriores que caracterizan la competencia lingüŕstica en términos formales y como representaciones mentales, es decir, con exclusivo carácter psicológicas. Sin embargo, Chomsky asume que la competencia gramatical puede ser caracterizada desde el punto de vista abstracto, es decir, computacionalrepresentacional y en términos de procesos neuroanatómicos o físicos, y ambos enfoques no son contradictorios, sino complementarios.

La adopción del enfoque naturalista por Chomsky implicó la total superación del dualismo mente/cuerpo. Es más, según el maestro del MIT, este dualismo carece de relevancia conceptual: Dado que el cerebro está involucrado en el estudio del lenguaje y en otros fenómenos mentales, se puede emplear el término 'mente' para referirnos al cerebro. En este sentido, el enfoque naturalista de la mente investiga los aspectos mentales del mundo, con la expectativa de una integración eventual con el «uúcleo» de las ciencias naturales (Chomsky 2002), por cuanto la facultad del lenguaje es un módulo o componente de la mente-cerebro y, por tanto, es de naturaleza física y neurofisiológica. 


\section{Labiolingürística}

Dado que Chomsky se adhiere a una concepción internalista en la explicación de la naturaleza del lenguaje, su enfoque puede verse como inmerso en el terreno de la biología: el lenguaje humano es un objeto biológico. Esta perspectiva abre paso a un horizonte que puede denominarse propiamente biolingüŕstica.

En concordancia con esta visión, el lenguaje debe ser analizado con la metooblogía de las ciencias naturales. Así, la lingüística puede asumirse como una ciencia natural y operar con el método de la idealización propio de las llamadas ciencias duras. Sin embargo, ello no implica una reducción del lenguaje a la física o biología, puesto que el lenguaje tiene sus propias leyes y generalizaciones que no pueden describirse en términos de las partículas o las neuronas. Esta intenpretación sí implica la unificación (no la reducción) de las ciencias, en la medida en que se aplique el mismo método en los diversos campos. Debemos decir que esta unificación ya era planteada por Chomsky desde 1979.

En este sentido el naturalismo es central al trabajo de Chomsky y explícitamente excluye las propuestas dualistas a favor de la concepción naturalista. Según la concepción naturalista, el lenguaje es un objeto biológico, esto es, tiene una naturaleza neuroanatómica. Ahora bien, debemos aclarar con énfasis que la recusación del dualismo no implica una mirada reduccionista, ya que un análisis sintáctico (por ejemplo, en términos de parsers) guarda una gran especificidad respecto de los anál isis químicos o biológicas. Ia tesis de la unidad de la ciencia debe distinguirse nítidamente de la tesis de la reducción de la ciencia. Es más, la primera es plausible; la segunda conduce a un callejón sin salida.

\section{Ell uso del lenguaje y las condiciones de interface}

Otro de los problemas centrales que aún permanece como uno de los misterios de la lingüristica es el abordaje del uso del lenguaje dentro del enfoque naturalista en la línea de la interpretación intemalista. Es verdad que casi por cincuenta años, la lingüristica chomskiana se ha orientado primordialmente al estudio de la competencia (esto es, el lenguaje-I), pero no ha dejado de llamarle la atención cómo la 
competencia se pone en uso en la performance, fenómeno altamente complejo que forma parte aún del conjunto de misterios del entendimiento del lenguaje.

Si analizamos la evolución de la teoría gramatical, no es posible negar que ha habido un progreso en comprender cómo los seres humanos procesan las oraciones que escuchan. Es más, se ha avanzado gracias al desarrollo de los siguientes aspectos que han aportado algún tipo de entendimiento sobre este tópico:

a Estudios experimentales y teóricos de la percepción del lenguaje y su prootucción.

ld Comprensión de la adquisición y el cambio de las lenguas.

ф Análisis de la función del cerebro en sujetos normales y en casos patológicos.

Además, existe una comprensión preliminar sobre cómo interpretamos emisiones en el contexto (el dbjetivo de la pragmática) , pero estamos my lejos de contar con un avance significativo sobre el tema.

El abordaje del uso del lenguaje puede entenderse postulando que la facultad del lenguaje está dentro de la arquitectura más amplia de la mente/cerebro. Es decir, se establecen las relaciones entre la facultad del lenguaje y otros sistemas cognitivos de la mente-cerebro. En la explicación de este fenómeno se sostiene que el uso del lenguaje es posible debido a la relación del estado lingüístico con los demás módulos de la performance. Esta relación se efectúa mediante la lectura de las estructuras generadas por el estado lingüístico, a través de dos niveles de interface: la forma fonética y la representación semántica (lo que, en términos técnicos, se denomina forma lógica) . La forma fonética es leída por el sistema sensorio-motor y la representación semántica por el sistema conceptual (de naturaleza intensional) y otros sistemas de pensamiento y acción.

En general, el fenómeno de la intenpretación de las representaciones del móotulo gramatical por otros módulos se conoce con el nombre de condiciones de legibilidad. Estas condiciones imponen ciertas restricciones a la facultad del lenguaje para que los sistemas de la performance puedan leer lo que genera el móoulo gramatical de la mente-cerebro. En particular, se da cuenta de cómo el hablante es capaz de interpretar ciertas cadenas (el principio de la interpretación 
plena) , y cómo hay cadenas curya interpretación no puede converger, esto es, son cadenas ilegibles.

\section{Conclusiones}

1. Por más de un siglo el lenguaje fue considerado esencialmente como un medio de comunicación y la lingüŕstica formó parte de los planes curriculares de las facultades de las ciencias sociales. consecuentemente, el objeto de la lingüristica era el comportamiento verbal y la lingüŕstica (en la visión conductista) se concebía como una disciplina antimentalista.

2. En este contexto irrumpió la revolución cognitivista (1950) que implicó un cambio de perspectiva: del estudio del comportamiento y sus productos (por ejemplo, textos) se pasó, con el enfoque generativo, al estudio de los mecanismos internos (de naturaleza mental) que participan en el pensamiento y la acción.

3. En los últimos 20 años, especialmente en Chomsky (2002) , la teoría generativa optó por el enfoque natural ista y la dirección intemalista. Este importante viraje implicó:

a La hipótesis de la disposición de una facultad del lenguaje que constituye un estado inicial en la mente/cerebro.

ld Como un «órgano del lenguaje» en el mismo sentido de los órganos del cuerpo (vista, sistema circulatorio, etc.) y en cuanto órgano del cuerpo, el lenguaje está genéticamente determinado.

4 Hasta la década del 70, la facultad del lenguaje se concebía como de naturaleza psicológica, y se asumía que la lingüística debía ser parte de la psicología. A partir de la década de 1980, se radicaliza la posición naturalista de Chomsky en el sentido de que la facultad del lenguaje constituye un órgano de la mente/cerebro. Por tanto, el lenguaje se concibe como un objeto biológico y, por ello, como parte de la biología humana. En consecuencia, debe ser objeto de una disciplina denominada «biolingüristica» (Chomsky 2002 y Jenkins 1999) . El tópico es el particular estado de los cerebros de la gente: el estado lingüŕstico. Ia biolingüŕstica busca elucidar su naturaleza, sus propiedades, su desarrollo y su variedad, y su fundamento en una dotación biológica innata. Este enfoque biolingüístico radicaliza, pues, la consideración intemalista del lenguaje. 
5. La propuesta del lenguaje como objeto de las ciencias naturales (biología, neurología) no constituye, sin embargo, un intento de reducir el lenguaje a la fisiología o a la física. Lo que se busca es integrar nuestras teorías de la mente (especialmente, la lingüística) con las teorías del cerebro y otros del daminio relevante.

6. El establecimiento de la facultad del lenguaje como estado de la mente/cerebro y como objeto de la lingüística, conduce a la interrogante de cuáles son las relaciones entre la facultad del lenguaje y los otros sistemas de la mente/cerebro. Se ha tratado de responder a ella sosteniendo que las operaciones de la facultad del lenguaje están reguladas por condiciones impuestas desde fuera, que se han denominado condiciones de legibilidad. Son condiciones impuestas por la necesidad que tienen otros sistemas de la mente/ cerebro para el uso de las representaciones provistas por la facultad del lenguaje. En ese sentido, la facultad del lenguaje participa en forma crucial en cada aspecto de la vida humana, del pensamiento y la interacción social.

7. Sólo una concepción intemalista del conocimiento del lenguaje es capaz de proveer una explicación de nuestras habilidades y el uso del lenguaje tanto en su producción como en su percepción.

8. Desde 1976, Chosmky divide el conocimiento del lenguaje en «problemas» $\mathrm{y}$ «misterios». Los problemas pueden ser elucidados mediante la teorización, pero no los «misterios». Ahora bien, dado que muchos misterios son sumamente importantes, el progreso en la ciencia lingüística consiste en transformar los misterios en problemas científicamente abordables. Todo indica que lo último está acaeciendo con el misterio del uso del lenguaje que podría ser abordado científicamente en términos de las condiciones de interface dentro de la arquitectura compleja de la mente-cerebro.

\section{Nota}

* Una versión anterior de este texto fue leída en el Seminario Taller de Investigación Femando Tola, llevado a cabo por la Facultad de Letras y Ciencias Humanas (octubre de 2005) . Constituye un esfuerzo por dar cuenta del desarrollo de la teoría lingüística en las últimas décadas y busca evaluar las altemativas de explicación sobre la naturaleza de las lenguas naturales. Chomsky (2002) y Bunge (1983) han sido las bases que han estimulado este cometido; por tanto, sus ideas están presentes en las diferentes secciones de este trabajo. 


\section{Referencias bibliográficas}

BUNGE, Mario (1983) : Lingüística y Filosofía. Editorial Ariel, Barcelona. CHOMSKY, Noam (1965) : Aspects of the Theory of Syntax. MIT Press, Cambridge, Mass.

CHOMSKY, Noam (1976): Reflections on Language. Pantheon, New York. CHOMSKY, Noam (1980) : Rules and Representations. Columbia University Press, New York.

GHOMSKY, Noam (1986) : Knowledge of Language: Its Nature, Origins and Use. Praeger, New York.

CHOMSKY, Noam (1988) : El lenguaje y los problemas del conocimiento. Editorial Visor, Medrid.

CHOMSKY, Noam (1995) : The Minimalist Program. MLT Press, Cambridge MA.

CHOMSKY, Noam (1998) : Nuestro conocimiento del lenguaje humano. Ediciones Universidad de Concepción \& Bravo y Allende, Concepción. CHOMSKY, Noam (2002) : New Horizons in the Study of Language and Mind. Cambridge University Press, Cambridge, UK.

EDEIMAN, Gerald M. (1992) : Bright Air, Brilliant Fire. On the Matter of Mind. Basic Books, New York.

JACOBSEN, Bent (1986) : Modem Transformational Grammar. Elsevier Science Publishers, North-Holland.

JENKINS, Lyle (1999) : Biolinguistics: Exploring the Biology of Language. Cambridge University Press, Cambridge UK.

KuHN, Thomas S. (1962) : The Structure of Scientific Revolutions. University of Chicago Press, Chicago.

LFNNEBERG, Eric H. (1967) : Biological Foundations of Language. Wiley, New York.

LORENZO, Guillermo (2001) Comprender a Chomsky. A. Machado Libros, Madrid.

MARCUSHI, Luiza y María M. Martins Salamão (2004) . En MUSSALIM, Femanda y Anna Christina Bentes (2004) Introoução à lingüística. Cortez, São Paulo.

SAUSSURE, Ferdinand de (2002) : Écrits de linguistique générale. Éditions Gellimard, Paris.

SIRAWSON, Gallen. (1997) : La realidad mental. Prensa Ibérica, Barcelona. 